\title{
RESIDUE CURRENTS AND COMPLEXITY PROBLEMS
}

\author{
M. ELKADI and A. YGER \\ Département de Mathématiques, Université Bordeaux 1 \\ 33405 Talence, France
}

1. An introduction: about Macaulay theorem. Let $P_{1}, \ldots, P_{m} m$ homogeneous polynomials in $n+1$ variables $\left(X_{0}, \ldots, X_{n}\right)$ with coefficients in a subfield $\mathbb{F}$ of $\mathbb{C}$ and respective degrees $D_{1}, \ldots, D_{m}$; assume further that $m \leq n+1$ and that the projective variety $\left\{P_{1}=\ldots=P_{m}=0\right\}$ is $n-m$ dimensional in $\mathbb{P}^{n}(\mathbb{C})$. The Chow form of the homogeneous ideal $\left(P_{1}, \ldots, P_{m}\right)$ is defined as follows: if one takes $n+1-m$ systems of parameters $U^{(1)}, \ldots, U^{(n+1-m)}$ in $\mathbb{C}^{n+1}$, the set of polynomials $R$ in $\left(U^{(1)}, \ldots, U^{(n+1-m)}\right)$ with coefficients in $\mathbb{F}$ such that $R$ times some power of the maximal ideal $\mathcal{M}=\left(X_{0}, \ldots, X_{n}\right)$ in $\mathbb{C}\left[X_{0}, \ldots, X_{n}\right]$ is included in

$$
\left(P_{1}, \ldots, P_{m},\left\langle U^{(1)}, X\right\rangle, \ldots,\left\langle U^{(n+1-m)}, X\right\rangle\right)
$$

is a principal ideal in $\mathbb{F}\left[U^{(1)}, \ldots, U^{(n+1-m)}\right]$; a generator for this ideal is a Chow form (or an eliminating form in the terminology of [Ph1]) for the ideal $I$. The Chow form plays an important role in arithmetic intersection theory: it can be used to "measure" the arithmetic complexity of the complete intersection cycle $\mathcal{D}_{1} \cap \ldots \cap \mathcal{D}_{m}$, where the $\mathcal{D}_{j}$ correspond to the algebraic hypersurfaces $\left\{P_{j}=0\right\}$. For example, if $\mathbb{F}=\mathbb{Q}$, one defines, as in [Ph4], the global height of $I$ as

$$
h(I)=\sum_{p \text { prime }} \ln M_{v_{p}}(\Phi)+\int_{\left\{\left\|U^{(1)}\right\|=1\right\}} \ldots \int_{\left\{\left\|U^{(n+1-m)}\right\|=1\right\}} \ln |\Phi(U)| d \sigma(U)
$$

where $\Phi$ is an eliminating form for $I, M_{v}(\Phi)$ denotes the maximum of the $v$-adic absolute values of all coefficients of $\Phi$, and $d \sigma$ is the differential form corresponding to the product of normalized Lebesgue measures on unit spheres $\left\{\left\|U^{(j)}\right\|=1\right\}, j=$ $1, \ldots, n-m+1$. The Chow form (or some power of it) appears as a denominator for the rational functions in $U$ involved in the expression of multidimensional residues. In fact, if $k=\left(k_{1}, \ldots, k_{m}\right) \in \mathbb{N}^{m}\left(\mathbf{1}^{\prime}\right.$ if all $k_{j}$ are equal to 1$)$ and

1991 Mathematics Subject Classification: 32A25, 32A27, 32C30, 14C17, 14Q20.

The paper is in final form and no version of it will be published elsewhere. 
$l=\left(l_{1}, \ldots, l_{n-m+1}\right) \in \mathbb{N}^{n-m+1}\left(\mathbf{1}^{\prime \prime}\right.$ if all $l_{j}$ are 1$)$, one can see, as a consequence of the transformation law ([GH], p. 657-659) and an algorithm by Kytmanov ([Ky], p. 495, formula 3) that for any test function $\varphi$ in $\mathcal{D}\left(\mathbb{C}^{n+1}\right)$ and almost any values $U^{(1)}, \ldots, U^{(n-m+1)}$ of the parameters $U$,

$$
\left\langle\bar{\partial}\left(\frac{1}{P^{*\left(k+\mathbf{1}^{\prime}\right)}}\right) \wedge \bar{\partial}\left(\frac{1}{\langle U, \zeta\rangle^{*\left(l+\mathbf{1}^{\prime \prime}\right)}}\right), \varphi d \zeta\right\rangle=\sum_{\substack{r \in \mathbb{N}^{n+1} \\ r \leq\left\langle k+\mathbf{1}^{\prime}, D\right\rangle+|l|-m}} R_{r}^{(k, l)}(U) \frac{\partial^{|r|}}{\partial \zeta^{r}} \varphi(0)
$$

where the $R_{r}^{(k, l)}$ are rational functions in $U$ with common denominator a power of the Chow form $\Phi$, namely $\Phi^{s(k, l)}$, where $s(k, l)=|k|+|l|+n+1$. To make things more explicit, let us write down the $n+1$ relations

(1) $\quad z_{k}^{q_{k}} \Phi(U)$

$$
=\sum_{j=1}^{m} A_{k, j}(Z, U) P_{j}(Z)+\sum_{l=1}^{n-m+1} A_{k, m+l}(Z, U)\left\langle U^{(l)}, Z\right\rangle, \quad k=0, \ldots, n .
$$

which are derived from the definition of the Chow form. Let $\Delta$ be the determinant of the $n+1, n+1$ matrix of the $A_{k, l}$. In order to simplify notations, given $(k, l) \in \mathbb{N}^{n+1}$, denote $t=t_{k, l}=|k|+|l|, \tau=\tau_{k, l}=\left(k+\mathbf{1}^{\prime}\right) !\left(l+\mathbf{1}^{\prime \prime}\right) ! ;$ if $\left(r_{1}, \ldots, r_{t}\right)$ is any element in $\mathbb{N}^{t}$, we denote $c\left(r_{1}, \ldots, r_{t}\right)$ the number $i_{0} ! \ldots i_{n}$ !, where $i_{j}$ (for $j=0, \ldots, n)$ is the number of times $\mathrm{j}$ appears in the sequence $r_{1}, \ldots, r_{t}$. We note also $q(r)$ the $n+1$-uplet $\left(q_{0}\left(i_{0}+1\right), \ldots, q_{n}\left(i_{n}+1\right)\right)$ and

$$
S_{k, l, r}=\prod_{\substack{1 \leq j \leq m \\ k_{1}+\ldots+k_{j-1}<\xi \leq k_{1}+\ldots+k_{j}}} A_{r_{\xi}, j} \prod_{\substack{1 \leq j \leq n+1-m \\|k|+l_{1}+\ldots+l_{j-1}<\xi \leq|k|+l_{1}+\ldots+l_{j}}} A_{r_{\xi}, m+j}
$$

with the convention $k_{0}=l_{0}=0$. Then, one can write

$$
\begin{array}{r}
\tau_{k, l}(\Phi(U))^{|k|+|l|+n+1}\left\langle\bar{\partial}\left(\frac{1}{P^{*\left(k+\mathbf{1}^{\prime}\right)}}\right) \wedge \bar{\partial}\left(\frac{1}{\langle U, \zeta\rangle^{*\left(l+\mathbf{1}^{\prime \prime}\right)}}\right), \varphi d \zeta_{0} \wedge \ldots \wedge d \zeta_{n}\right\rangle \\
=\sum_{0 \leq r_{1}, \ldots, r_{t} \leq n} \frac{c\left(r_{1}, \ldots, r_{t}\right)}{(q(r)-\mathbf{1}) !}\left[\frac{\partial^{|q(r)|}}{\partial \zeta^{* q(r)}}\left(\varphi \Delta(\cdot, U) S_{k, l, r}(\cdot, U)\right)\right](0) .
\end{array}
$$

As seen above, we will always use the multiplicative formal notations for residue currents: if $f_{1}, \ldots, f_{p}$ denote $\mathrm{p}$ functions and $t=\left(t_{1}, \ldots, t_{p}\right)$ is a $p$-uplet of positive integers,

$$
\bar{\partial}\left(1 / f^{* t}\right)=\bar{\partial}\left(1 / f_{1}^{t_{1}}\right) \wedge \ldots \wedge \bar{\partial}\left(1 / f_{p}^{t_{p}}\right) .
$$

A fundamental result of Macaulay [Mac] states that, when $m=n+1$, and $P_{1}, \ldots, P_{n+1}$ define the origin in $\mathbb{C}^{n+1}$, then, for $\kappa=D_{1}+\ldots+D_{n+1}-n$, the ideal $\mathcal{M}^{\kappa}$ is included in $\left(P_{1}, \ldots, P_{n+1}\right)$; an effective version of this fact can be obtained from a classical formula of Jacobi and gives some insight on the role played by multidimensional residues in division problems. Before stating this result, we need to introduce systems of Hefer divisors for $P_{1}, \ldots, P_{n+1}$, that is polynomials $g_{j, k}$, 
$j=1, \ldots, n+1, k=0, \ldots, n$ such that, for any $j$ in $\{1, \ldots, n+1\}$,

$$
P_{j}(\zeta)-P_{j}(Z)=\sum_{k=0}^{n} g_{j, k}(Z, \zeta)\left(\zeta_{k}-z_{k}\right)
$$

The result of Macaulay can be written as:

TheOREM 1. Any monomial $M$ of total degree $\rho \geq \kappa$ can be reproduced as

(2) $\quad M(Z)$

$$
=\sum_{\substack{k \in \mathbb{N}^{n+1},|k|>0 \\\langle k, D\rangle \leq \rho}}\left\langle\bar{\partial}\left(\frac{1}{P^{*(k+1)}}\right)(\zeta), M(\zeta) \bigwedge_{j=1}^{n+1}\left(\sum_{k=0}^{n} g_{j, k}(Z, \zeta) d \zeta_{k}\right)\right\rangle P(Z)^{* k} .
$$

Sketch of proof. The proof of this theorem is very simple; it involves two basic ingredients which seem to play a crucial role in division problems in commutative algebra. One is the Cauchy-Weil formula which gives some semilocal representation formula for $M$ in a neighborhhood of the origin in $\mathbb{C}^{n+1}$; this neighbourhood will be of the form

$$
\left\{\left|P_{1}(\zeta)\right|<\epsilon_{1}(\xi) \sim 1 / \xi^{D_{1}}, \ldots,\left|P_{n+1}(\zeta)\right|<\epsilon_{n+1}(\xi) \sim 1 / \xi^{D_{n+1}}\right\}=\Omega_{\xi}, \xi \gg 0 .
$$

Cauchy-Weil's formula [Weil] provides in $\Omega_{\xi}$ the following:

$$
\begin{aligned}
M(Z)= & \frac{1}{(2 i \pi)^{n+1}}\left(\int_{\Gamma_{\xi}} \frac{M \bigwedge_{j=1}^{n+1}\left(\sum_{k=0}^{n} g_{j, k}(Z, \zeta) d \zeta_{k}\right)}{\prod_{j=1}^{n+1}\left(P_{j}(\zeta)-P_{j}(Z)\right)}\right. \\
& \left.-\int_{\Gamma_{\xi}} M \frac{\bigwedge_{j=1}^{n+1}\left(\sum_{k=0}^{n} g_{j, k}(Z, \zeta) d \zeta_{k}\right)}{\prod_{j=1}^{n+1} P_{j}}\right)
\end{aligned}
$$

( $\Gamma_{\xi}$ is the Shilov boundary of $\Omega_{\xi}$ and the second integral in the right-hand side above is 0 since the total degree of $M$ is larger than $\kappa$, which corresponds to the fact that $\mathcal{M}^{\kappa}$ is included in $\left.\left(P_{1}, \ldots, P_{n+1}\right)\right)$. We now expand the kernels in the integrals as the Cauchy kernel in Cauchy formula, which gives in $\Omega_{\xi}$ the following semi local division formula:

$$
M(Z)=\sum_{k \in \mathbb{N}^{n+1},|k|>0}\left\langle\bar{\partial}\left(\frac{1}{P^{*(k+\mathbf{1})}}\right)(\zeta), M(\zeta) \bigwedge_{j=1}^{n+1}\left(\sum_{k=0}^{n} g_{j, k}(Z, \zeta) d \zeta_{k}\right)\right\rangle P^{* k}(Z)
$$

The series in the above formula is in fact truncated, due to the second key argument of the proof, that is Jacobi's formula ([Jac], $[\mathrm{GH}]$ ) which states (as a consequence of the global residue formula on the compact variety $\mathbb{P}^{n}(\mathbb{C})$ ) that for any polynomial $S$,

$$
\left\langle\bar{\partial}\left(\frac{1}{P^{*(k+1)}}\right)(\zeta), S(\zeta) d \zeta_{0} \wedge \ldots \wedge d \zeta_{n}\right\rangle=0
$$

as soon as $\langle k+\mathbf{1}, D\rangle \geq \operatorname{deg}(S)+n+2$. 
In the same vein, we have the following representation formula in order to make explicit the membership of some homogeneous polynomial $Q$ in the homogeneous ideal generated in $\mathbb{C}\left[\zeta_{0}, \ldots, \zeta_{n}\right]$ by $\left(P_{1}, \ldots, P_{m}\right)$ (defining a complete intersection in $\left.\mathbb{P}^{n}(\mathbb{C})\right)$. Given $n-m+1 n+1$-uplets of parameters $U^{(1)}, \ldots, U^{(n-m+1)}$ in $\mathbb{C}^{n+1}$, we will denote by $\Psi(U)$ the differential form

$$
\Psi(U)=\bigwedge_{j=1}^{n-m+1}\left(\sum_{k=0}^{n} U_{k}^{(j)} d \zeta_{k}\right), \quad U^{(j)}=\left(U_{0}^{(j)}, \ldots, U_{n}^{(j)}\right)
$$

In order to make things clearer, we will denote by $G$ the differential form associated to systems of Hefer divisors for $P_{1}, \ldots, P_{m}$,

$$
G(Z, \zeta)=\bigwedge_{j=1}^{m}\left(\sum_{k=0}^{n} g_{j, k}(Z, \zeta) d \zeta_{k}\right)
$$

Then, we have the following result:

THEOREM 2. Let $Q$ be a homogeneous polynomial of degree $d$ in $n+1$ variables which lies in the complete intersection ideal $\left(P_{1}, \ldots, P_{m}\right)$; then, for any $Z$ in $\mathbb{C}^{n+1}$,

(3) $Q(Z)=\sum_{s=1}^{m}\left(\sum_{\substack{\mathcal{S} \subset[1, m] \\ \# \mathcal{S}=s}}\left(\sum_{\substack{k \in \mathbb{N}^{m} \\\langle k, D\rangle+|l| \mathbb{N}^{n-m+1} \\\left\langle k, \sum_{j \in \mathcal{S}} D_{j} \leq d\right.}} c_{\mathcal{S}}^{k, l} P^{* k}(Z)\langle U, Z\rangle^{* l}\right) \prod_{j \in \mathcal{S}} P_{j}(Z)\right)$

where, for any $\mathcal{S} \subset\{1, \ldots, m\}$, any $k$ in $\mathbb{N}^{m}$ and any $l$ in $\mathbb{N}^{n-m+1}$,

$c_{\mathcal{S}}^{k, l}=\left\langle\bar{\partial}\left(\frac{1}{P^{*\left(k+\mathbf{2}^{\prime}\right)}}\right) \wedge \bar{\partial}\left(\frac{1}{\langle U, \zeta\rangle^{*\left(l+\mathbf{1}^{\prime \prime}\right)}}\right), Q\left(\prod_{j \notin \mathcal{S}}\left(P_{j}-P_{j}(Z)\right)\right) G(Z, \zeta) \wedge \Psi(U)\right\rangle$.

Proof. The proof of this result is based on the same two key points as before; additionally, we profit from the fact that Weil's formula, even written for a system $\left(f_{1}, \ldots, f_{n+1}\right)$ of $n+1$ holomorphic functions defining a discrete variety in some open subset of $\mathbb{C}^{n+1}$, can be used to reproduce the membership of some holomorphic function $\mathrm{f}$ in the ideal generated by $m$ of the $\mathrm{f}_{j}$ 's (see for example [BT], section 1).

In the statement of theorem 2, the coefficients of the $c_{S}^{k, l}$ (considered as polynomials in $Z$ ) are rational functions in $U$ with, as a denominator, some power of the Chow form of the ideal (the exponent being at most $|k|+|l|+n+1$ if one uses Kytmanov's theorem ([Ky], p. 495, formula (3)). It appears in this statement that the effectivity of division problems for the ideal $\left(P_{1}, \ldots, P_{m}\right)$ is controlled by the list $\mathcal{R}$ of rational functions $R_{r}^{k, l}, k \in \mathbb{N}^{m}, l \in N^{n-m+1}, r \leq\left\langle k+\mathbf{1}^{\prime}, D\right\rangle+|l|-m$ already introduced above. If one thinks about an arithmetic setting (imagine for example that the $P_{j}$ have integer coefficients and that the Chow form is an integral Chow form in the sense of [Br2]), then we have a countable list of elements in $\mathbb{Z}\left(U^{(1)}, \ldots, U^{(n-m+1)}\right)$ which gives us some explicit control for bounds in the membership problem. The denominators involved in all these rational functions 
are powers of some eliminating form for the ideal with integer coefficients; we already mentioned before that such a form could be used to measure the arithmetic complexity of the ideal (or of the corresponding cycle) in arithmetic intersection theory. In fact, any information on the Chow form (which controls intersection theory), such as a bound for its degree or its height, appears as some information for the denominators in this list $\mathcal{R}$ of rational functions (which controls division theory). This is the main point we would like to derive from this approach of Macaulay's theorem. In order to obtain estimates for the numerators involved in the rational functions involved in (1) when the $P_{j}$ have integer coefficients, one can take advantage of the following fact: the polynomial

$$
\left.\Phi(U)^{|k|+|l|+n+1} \tau_{k, l}\left(\prod_{j=0}^{n}\left(q_{j}(|k|+|l|+1)-1\right)\right) !\right) R_{r}^{k, l}(U)=T_{r}^{k, l}(U)
$$

is in $\mathbb{Z}[U]$. The estimates on the degree of this polynomial follow from the fact that the total degrees (with respect to each block of variables $U^{(j)}$ ) of $\Phi$ and of the $A_{j, k}$ in (1) (as polynomials in $U$ ) are bounded by $D_{1} \ldots D_{m}$. Therefore, to get any bound on the coefficients of this polynomial (and therefore obtain some control on the size of quotients in division problems), it is enough to get some analytic estimate for $T_{r}^{k, l}(U)$; such an estimate will follow from two facts: first, from $E o$ jasiewicz Nullstellen inequalities (see theorem 2 in [Br3]), there exists some polynomial with integer coefficients $\delta(U)$ and some constant $\kappa$ well estimated such that

$$
\operatorname{Max}\left|P_{j}(\alpha)\right|_{p r}+\operatorname{Max}\left|\left\langle U^{(l)}, \alpha\right\rangle\right|_{p r} \geq \frac{1}{\kappa}|\delta(U)|, \alpha \in\left(\mathbb{C}^{n+1}\right)^{*} ;
$$

then, from the Bochner-Martinelli formula, we have, if $\epsilon=(-1)^{n(n-1) / 2}$,

$$
R_{r}^{k, l}(U)=\epsilon \frac{n !(n+|k|+|l|) !}{(2 i \pi)^{n+1} k ! l !} \operatorname{Inf}_{R>0}\left(\int_{\{\|\zeta\|=R\}} \frac{\bar{f}^{*(k, l)} \sum_{j=1}^{n+1}(-1)^{j-1} \bar{f}_{j} d \bar{f}_{[j]} \wedge \zeta^{r} d \zeta}{\|f\|^{2(n+1+|k|+|l|)}}\right)
$$

where $f=f_{U}=\left(P_{1}, \ldots, P_{m},\left\langle U^{(1)}, \zeta\right\rangle, \ldots,\left\langle U^{(n-m+1)}, \zeta\right\rangle\right)$. Though $\delta$ does not seem explicit, one may get some control on the size of the integer coefficients involved in some identity

$$
a Q=\sum_{j=1}^{m} Q_{j} P_{j}, \quad a \in \mathbb{Z}, \quad Q_{j} \in \mathbb{Z}\left[X_{0}, \ldots, X_{n}\right]
$$

valid for some homogeneous polynomial $Q$ in $\left(P_{1}, \ldots, P_{m}\right)$, where $P_{1}, \ldots, P_{m}$ are homogeneous polynomials with integer coefficients defining a complete intersection in $\mathbb{P}^{n}(\mathbb{C})$. The idea is to choose systems of integer parameters $\left(U^{(j)}\right)$ in order that $|\Phi(U)| \neq 0$ (bounds on these precise parameters are given by zero lemmas) and to use Bochner-Martinelli formula combined with Nullstellen inequalities (see [Ph2] or [Br2]) in order to get estimates for the numerators of the $R_{r}^{k, l}$ and therefore for the integer coefficients of some solution $\left(Q_{1}, \ldots, Q_{m}\right)$ involved in (5). Most of the ideas we will develop to obtain results about effectivity problems in the affine case (see the next sections) are based on the same ideas. 
2. Some effectivity results in the affine case. Sharp bounds for the degrees in the resolution of the Bézout identity or the membership problem for ideals have been obtained, first from a combination of transcendental methods (namely the theory of elimination via Chow forms, see $[\mathrm{HP}]$ ) and analytic arguments (i.e Skoda's theorem $[\mathrm{Sk}]$ which, given polynomials $P_{1}, \ldots, P_{m}$ in $\mathbb{C}\left[X_{1}, \ldots, X_{n}\right]$ and some polynomial $Q$ which is at every point $\alpha$ in $\mathbb{C}^{n}$ locally in the integral closure of $\left(\left(P_{1}\right)_{\alpha}, \ldots,\left(P_{m}\right)_{\alpha}\right)$, provides some explicit way (based on $L^{2}$ estimates ) in order to write $Q^{\inf (n, m)}$ in $\left(P_{1}, \ldots, P_{m}\right)$. Of course, Skoda's theorem, to be efficient, needs that one has some a priori growth estimates for the locally constant function $C$ such that

$$
|Q(Z)| \leq C(Z)\left(\sum_{j=1}^{m}\left|P_{j}(Z)\right|^{2}\right)^{1 / 2} .
$$

When the $P_{j}$ have no common zeroes, such estimates were given by D. Brownawell in [Br1] and one can find polynomials $Q_{j}$ with total degree $n \mu D_{1} \ldots D_{\mu}+\mu D_{1}$ $\left(D_{1} \geq \ldots \geq D_{m}, \mu=\inf (n, m)\right)$ such that

$$
1=\sum_{j=1}^{m} P_{j} Q_{j}
$$

which is the so called Bézout identity.

Of course, when the projective variety associated to $P_{1}, \ldots, P_{m}$ is empty, much better bounds are known (see Macaulay's theorem in section 1). The difficulty, from the point of view of algebraic geometry, is to get some control on the embedded components of the homogeneous ideal generated by $\left({ }^{h} P_{1}, \ldots,{ }^{h} P_{m}\right)\left({ }^{h} P\right.$ is the homogenized version of $P$ ); the isolated components do not contribute to any trouble because of Bézout intersection theorem. In fact, this difficulty was cleared by J. Kollár in $[\mathrm{Ko}]$; the latest result relative to degree estimates in the Nullstellensatz was stated by D. Brownawell [Br4], following Kollár's argument.

THEOREM 3. Let $\mathbb{K}$ be an arbitrary field and $I$ an ideal in $\mathbb{K}\left[X_{1}, \ldots, X_{n}\right]$ generated by $m$ polynomials $P_{1}, \ldots, P_{m}$, with $\operatorname{deg}\left(P_{j}\right) \leq D_{j}$ and $D_{2} \geq \ldots \geq$ $D_{m} \geq D_{1}>0, D_{m} \geq 3$. If $\mathcal{Q}_{1}, \ldots, \mathcal{Q}_{r}$ are the prime minimal ideals in the decomposition of $I$ in primary components, there are positive integers $e_{1}, \ldots, e_{r}$ such that

$$
\mathcal{Q}_{1}^{e_{1}} \ldots \mathcal{Q}_{r}^{e_{r}} \subset I, \quad e_{1}+\ldots+e_{r} \leq D_{1} \ldots D_{\mu}, \quad \mu=\inf (n, m) .
$$

As a consequence of this result, one can show that a sharp bound for the degrees of the $Q_{j}$ in (7) (when the $P_{j}$ have no common zero) is precisely $D_{1} \ldots D_{\mu}$ (with the same technical restrictions than in Theorem 3, still unavoidable at the present point). A classical telescopic example (see [Br1]) show that these bounds on the degrees are sharp. Since we know, from a classical example of Mayr-Meyer ([MaM], $[\mathrm{BaS}]$ ), that the membership problem for polynomial ideals has a doubly exponential complexity, it is interesting to give particular examples (apart from 
the classical Nullstellensatz, which appears to be equivalent to the problem of solving Bézout identity) where the problem can be solved in subexponential time.

One of them is the case of regular sequences; if $P_{1}, \ldots, P_{m}$ define a complete intersection in $\mathbb{C}^{n}$, and if $Q$ is in the ideal generated by the $\mathrm{P}_{j}$, there are polynomials $Q_{j}$ such that (7) holds, with

$$
\operatorname{Max}\left(\operatorname{deg}\left(Q_{j}\right)\right) \leq \operatorname{deg}(Q)+\kappa(n) D_{1} \ldots D_{m} .
$$

This was proved originally using a combination of transcendental methods and analytic ones ( $\bar{\partial}$ estimates) as in [BY1]; then, it was obtained from Kollár's argument by $\mathrm{F}$. Amoroso in [Am1]; in this latest work, under a restriction on the degrees $\left(D_{j} \geq 3\right)$, the constant $\kappa(n)$ disappears in the estimates (8).

Another interesting example where the membership problem can be solved in subexponential time is the case when the polynomials $P_{1}, \ldots, P_{m}$ define a smooth manifold in $\mathbb{C}^{n}[\mathrm{Am}]$; more recently, Amoroso proved in [Am4] that the membership problem is also solvable with exponential bounds when $P_{1}, \ldots, P_{m}$ define an "almost complete intersection" ideal: when there exist $k$ linear combinations $p_{1}, \ldots, p_{k}$ of the $P_{j}$ such that $\left(p_{1}, \ldots, p_{k}\right)=I \cap J$, where $I=\left(P_{1}, \ldots, P_{m}\right)$ and $J$ is an unmixed ideal with rank $k$ such that $I+J=\mathbb{C}\left[X_{1}, \ldots, X_{n}\right]$.

As we have already mentionned it, analytic methods are really adapted to the effectiveness of Briançon-Skoda theorem. If $Q$ is a polynomial such that there exists a global locally constant function $C$ such that

$$
|Q(Z)| \leq C(Z)\left(\sum_{j=1}^{m}\left\|P_{j}(Z)\right\|^{2}\right)^{1 / 2}
$$

there is an explicit division formula

$$
Q^{\mathrm{inf}(n, m)}=\sum_{j=1}^{m} P_{j} Q_{j} .
$$

We refer to [BGVY] for these formulas; the key point we should mention is that the degree estimates of the $Q_{j}$ (as well as their definition) involves some information about the distributions involved in the Laurent development about the origin of

$$
\lambda \longrightarrow\left(\sum_{j=1}^{m}\left|P_{j}\right|^{2}\right)^{\lambda-\sigma-1}, \quad \sigma=\inf (m, n+1)
$$

considered as a meromorphic valued function of $\lambda$, with values in $\mathcal{D}^{\prime}\left(\mathbb{C}^{n}\right)$ (see [At]). Very little is known at the moment about such distributions; nevertheless, there is a very new result (due to Amoroso in $[\mathrm{Am} 2]$, when $P_{1}, \ldots, P_{m}$ define a curve, or in [Am3] in general) which asserts the following: given $Q$ satisfying (9) with respect to polynomials $P_{1}, \ldots, P_{m}$ (with the same restrictions on the degrees than in the statement of theorem 3), there are polynomials $Q_{j}$ such that, if

$$
\eta(n)=\frac{3}{8}\left(3^{n}-1\right)+\frac{(n-1)^{2}}{4}+1
$$


then

$$
Q^{\eta(n)}=\sum_{j=1}^{m} Q_{j} P_{j}, \quad \operatorname{deg}\left(Q_{j} P_{j}\right) \leq \eta(n) \operatorname{deg}(Q)+D_{1}+D_{1} \ldots D_{\mu} .
$$

Of course, the exponent $3^{n}$ seems to be an avatar of Kollár's method, which at the moment provides this result. What is very curious here is that the exponent $\eta(n)$ does not depend on the degree of the $P_{j}$ (which is the case in the algebraic Nullstellensatz). This result, obtained by means of geometric methods, gives some indication that analytic methods should provide "economic formulas" with respect of degree bounds in algebraic division problems. We refer at the moment to [BY4] and [BY5] for some hints about how to attack such questions.

The problems which remain unsolved and not completely understood concern height estimates in division formulas. In the last section, we will explain in more detail the notion of height for arithmetic cycles in $\mathbb{P}^{n}=\operatorname{Proj}\left(\mathbb{Z}\left[X_{0}, \ldots, X_{n}\right]\right)$ which has been introduced by Faltings [Fa], Gillet-Soulé ([BGS1], [BGS2]); let us just mention that, when $\mathcal{P}$ is a prime homogeneous ideal in $\mathbb{Q}\left[X_{0}, \ldots, X_{n}\right]$ and $\mathcal{X}$ the corresponding arithmetic cycle in $\mathbb{P}^{n}$, then the height of $\mathcal{X}$ (in the sense of Faltings) differs from the global height of $\mathcal{P}$ (defined with respect of the Chow form of $\mathcal{P}$ in the sense of Philippon, as seen in section 1) by some quantity one can estimated by

$$
\kappa(n) \operatorname{deg}(\mathcal{X})
$$

(where $\operatorname{deg}(\mathcal{X})$ denotes the algebraic degree of $\mathcal{X}(\mathbb{C})$ in $\mathbb{P}^{n}(\mathbb{C})$ ). Arithmetic intersection theory provides an arithmetic version of Bézout theorem [BGS1], as follows: if $\mathcal{X}_{1}, \mathcal{X}_{2}$ are two arithmetic cycles in $\mathbb{P}^{n}$ of respective dimension in $\mathbb{P}^{n}$, $e_{1}+1, e_{2}+1$ which intersect properly, then

(10) $h\left(\mathcal{X}_{1} \cap \mathcal{X}_{2}\right) \leq h\left(\mathcal{X}_{1}\right) \operatorname{deg}\left(\mathcal{X}_{2}\right)+h\left(\mathcal{X}_{2}\right) \operatorname{deg}\left(\mathcal{X}_{1}\right)+\kappa\left(e_{1}, e_{2}\right) \operatorname{deg}\left(\mathcal{X}_{1}\right) \operatorname{deg}\left(\mathcal{X}_{2}\right)$

where $\kappa\left(e_{1}, e_{2}\right)=\inf \left(\theta\left(e_{1}\right), \theta\left(e_{2}\right)\right)$, and

$$
\theta(d)=-\frac{1}{2}\left(\sum_{k=1}^{d} \sum_{j=1}^{k} \frac{1}{j}+\sum_{k=1}^{n-d-1} \sum_{j=1}^{k} \frac{1}{j}\right)+\frac{n+1}{2} \ln (2) .
$$

This is the key reason why one can get estimates for denominators in the solution of the Bézout identity: if $P_{1}, \ldots, P_{m}$ are polynomials with integer coefficients without any common zero in $\mathbb{C}^{n}$, there exist polynomials $Q_{1}, \ldots, Q_{m}$ with integer coefficients and some non-zero integer a such that

$$
a=\sum_{j=1}^{m} P_{j} Q_{j}, \quad \ln (|a|) \leq \kappa(n) h D^{\sigma}\left(\frac{1}{h}+\sum_{j=1}^{\sigma} \frac{1}{D_{j}}\right)
$$

where $\sigma=\inf (m, n+1)$ and $h$ denotes the maximum of "naïve" logarithmic heights of the $P_{j}$, the naïve height $h(P)$ of $P \in \mathbb{Z}\left[X_{1}, \ldots, X_{n}\right]$ being defined as $\operatorname{Max}(\ln |\xi|)$, where the Max is taken over all non zero coefficients of $P$. The result we quote here is due to P. Philippon in [Ph3] and was obtained from the algebraic 
version of Briançon-Skoda theorem, that is Lipman-Teissier result [LT]: if $\mathcal{A}$ is a local regular ring with dimension $k$ and $I$ any ideal in $\mathcal{A}$, then, if $\bar{I}$ denotes the integral closure of $I$, one has $\bar{I}^{k} \subset I$. Unfortunately, if the bounds on the degrees of the $Q_{j}$ in $(11)$ are almost $\operatorname{sharp}\left(\operatorname{deg}\left(P_{j} Q_{j}\right)(n+2) D_{1} \ldots D_{\mu}, \mu=\inf (n, m)\right)$, the bounds for the naïve height of their coefficients are just the bounds provided by a linear algebra argument, that is $\sim \kappa(n) h\left(D^{n}\right)^{n}$; this does not fit with what we claimed in section 1 , that is that both intersection and division should be controled by the same tool, for example the multidimensional Grothendieck residue for regular sequences. Of course, we are far away from optimal results, but the first proposition we can get in this direction is the following:

Theorem 4 (Berenstein, Elkadi, Yger). Let $P_{1}, \ldots, P_{m}$ be $m$ polynomials with respective degrees $D_{1} \geq D_{2} \geq \ldots \geq D_{m}$ and coefficients in $\mathbb{Z}$; let $h$ be the maximum naïve logarithmic height of their coefficients; assume that the $P_{j}$ have no common zeroes in $\mathbb{C}^{n}$; there exists a positive integer a, some polynomials $Q_{1}, \ldots, Q_{m}$ in $\mathbb{Z}\left[X_{1}, \ldots, X_{n}\right]$ such that

$$
a=\sum_{j=1}^{m} P_{j} Q_{j}, \quad \operatorname{Max}_{j}\left(\operatorname{deg}\left(Q_{j}\right)\right) \leq n(2 n+1) D_{1} \ldots D_{\mu},
$$

$$
\operatorname{Max}\left(\ln |a|, h\left(Q_{j}\right)\right) \leq \kappa(n) D_{1}^{4}\left(D_{1} \ldots D_{\mu}\right)^{8}\left(h+\ln (m)+D_{1} \ln \left(D_{1}\right)\right) .
$$

This result, in its primitive form, was obtained in [BY2]; a careful look at the method (which has been simplified in [BY3], where appear the two key ingredients mentioned in section 1, that is Cauchy-Weil and Jacobi formulas) shows that one can separate the degrees as in the above statement; this was the contribution of M. Elkadi. We will not say anything here about the proof, just reminding that it consists mainly in plotting down a formula which is obtained as follows. First, choose $n$ linear combinations of the $P_{j}$ 's, $p_{1}, \ldots, p_{n}$ defining a system in normal position in $\mathbb{C}\left[X_{1}, \ldots, X_{n}\right]$ (every subsequence defines a complete intersection); this is the only non-explicit step in the construction of the formula. Then, find $n$ linear forms $L_{1}, \ldots, L_{n}$ with coefficients in $\mathbb{Z}$ such that, after a convenient change of variables, the columns of the matrix of these coefficients are in very steep geometric progression (taking into account a priori bounds depending on $h, m$ and the $\mathrm{D}_{j}$ 's). For any $N>1$, such linear forms satisfy

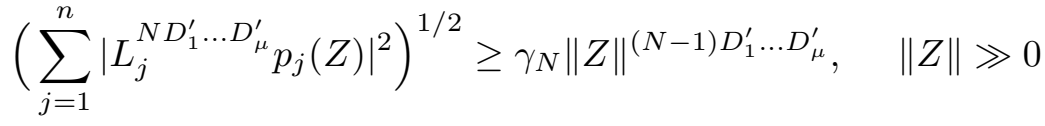

for some $\gamma_{N}>0$ (here $D_{j}^{\prime}=\max \left(D_{j}, 3\right)$ ). If $\left(g_{j, k}^{(N)}\right)_{1 \leq j, k \leq n}$ is a matrix of Hefer divisors for $L_{1}^{N \Delta} p_{1}, \ldots, L_{n}^{N \Delta} p_{n}\left(\Delta=D_{1}^{\prime} \ldots D_{\mu}^{\prime}\right)$, and $p_{n+1}$ a linear combination of the $P_{j}$ 's non vanishing on the set of common zeroes of $L_{1} p_{1}, \ldots, L_{n} p_{n}$, with $\left(g_{n+1, k}\right)_{1 \leq k \leq n}$ a system of Hefer divisors for $p_{n+1}$, we have, for $N$ large enough $\left(\sim c n^{2}\right)$ 


$$
\begin{aligned}
& 1=\left\langle\bar{\partial}\left(\frac{1}{L_{1}^{N \Delta} p_{1}}\right) \wedge \ldots \wedge \bar{\partial}\left(\frac{1}{L_{n}^{N \Delta} p_{n}}\right),\right. \\
& \left.\left|\begin{array}{ccccc}
g_{1,1}^{(N)}(Z, .) & . & . & . & g_{n+1,1}(Z, .) \\
\cdot & . & . & . & . \\
\cdot & . & . & . & . \\
g_{1, n}^{(N)}(Z, .) & . & . & . & g_{n+1, n}(Z, .) \\
L_{1}^{N \Delta} p_{1}(Z) & . & . & . & p_{n+1}(Z)
\end{array}\right| \frac{d \zeta_{1} \wedge \ldots \wedge d \zeta_{n}}{p_{n+1}}\right\rangle
\end{aligned}
$$

After clearing denominators, this formula provides the conclusion of the theorem.

In fact, the membership problem in the case of complete intersection can be solved using the same kind of arguments; the proof of the following result is similar to the proof of theorem 2 we gave in section 1 .

TheOREM 5 (M. Elkadi [Elk1]). Let $P_{1}, \ldots, P_{m}$ be $m$ polynomials with integer coefficients defining a complete intersection in $\mathbb{C}^{n} ;$ let $Q$ be a polynomial with integer coefficients lying in the ideal generated by the $P_{j}$ 's in $\mathbb{Q}\left[X_{1}, \ldots, X_{n}\right]$; there is an integer $a \in \mathbb{N}^{*}$, polynomials $Q_{1}, \ldots, Q_{m}$ in $\mathbb{Z}\left[X_{1}, \ldots, X_{n}\right]$ such that

$$
a Q=\sum_{j=1}^{m} Q_{j} P_{j}, \quad \operatorname{deg}\left(Q_{j}\right) \leq 2 \operatorname{deg}(Q)+\kappa(n, m) D_{1} \ldots D_{m},
$$

$\ln |a|, \ln \left(h\left(Q_{j}\right)\right.$

$$
\leq \kappa(n)\left[D_{1}^{4}\left(D_{1} \ldots D_{m}\right)^{5}\left(\operatorname{deg}(Q)+D_{1} \ldots D_{m}\right)^{3}\left(h+D_{1} \ln \left(D_{1}\right)\right)+h(Q)\right] .
$$

In fact, one has

$$
\kappa(n, m)=3 n(n+1)(2 n+1) m^{n}+3 m n+10 n+9 m-5 .
$$

The proof of this theorem is based as before on the construction of linear combinations of the $P_{j}$ 's defining a system in normal position; then, we introduce affine forms $L_{1}, \ldots, L_{n}$ such that, for any $N>1$, there exists a strictly positive constant $\gamma_{N}$ such that

$$
\left(\sum_{j=1}^{m}\left|L_{j}^{N\left(D_{1} \ldots D_{m}\right)} p_{j}(Z)\right|^{2}+\sum_{j=m+1}^{n}\left|L_{j}^{N\left(D_{1} \ldots D_{m}\right)}(Z)\right|^{2}\right)^{1 / 2} \geq \gamma_{N}\|Z\|^{(N-1) D_{1} \ldots D_{m}}
$$

for $\|Z\| \gg 0$. Once this is done, we recover the membership of

$$
\left(L_{1} \ldots L_{m}\right)^{3 D_{1} \ldots D_{m}} Q
$$

in the ideal generated by the $L_{j}^{3 \Delta} p_{j}, \Delta=D_{1} \ldots D_{m}$ with a formula derived from Weil's and Jacobi's formula as in section 1 . The conclusion of the theorem relies on the fact that one can use sufficiently many families (in fact $n+1$ ) of $n$ affine forms, in order that the different products of all forms in the same family have no common zeroes in $\mathbb{C}^{n}$. Once this is done, we use an easy Bézout identity for all these products (see all the details in [Elk2]). 
3. Conclusion; Green currents and intersection theory. In order to conclude this short survey, we would like to take the opportunity to mention how Green's equation $d d^{c}(T)=\delta_{X}$ (for $\mathcal{X}$ some analytic or algebraic cycle), or "half Green's equation", that is $\bar{\partial} T=R, R$ being some residue current relative to the ideal defining $\mathcal{X}$ (when it exists), plays some role in intersection (or division) theory even in an arithmetic context.

First, when $P_{1}, \ldots, P_{m}$ define a system in normal position, all currents which are involved in the quotients (if one refers to the analytic membership problem solution) $Q_{1}, \ldots, Q_{m}$ in $Q=\sum P_{j} Q_{j}$ are currents $T$ such that $\bar{\partial} T=\bar{\partial}(1 / f)$, where $f$ is a system of monomials in $\left(P_{1}, \ldots, P_{m}\right)$.

Parallelly, the equation that seems to be crucial in arithmetic intersection theory on $\mathbb{P}^{n}=\operatorname{Proj}\left(\mathbb{Z}\left[X_{0}, \ldots, Z_{n}\right]\right)$ is the following

$$
d d^{c}(T)+\delta_{\mathcal{X}}=\bigwedge_{j=1}^{m} c_{j}\left(\mathcal{D}_{j}\right)
$$

where the $c_{j}\left(\mathcal{D}_{j}\right)$ are the first Chern classes of the fiber bundles $\left[\mathcal{D}_{1}\right], \ldots,\left[\mathcal{D}_{m}\right]$. The $p$ arithmetic Chow group

$$
\left(C H^{p}\right)^{\wedge}\left(\mathbb{P}^{n}\right)
$$

is precisely defined as the quotient group of the group of Chow pairs $(\mathcal{X}, G)(\mathcal{X}$ being a codimension $p$ arithmetic cycle in $\mathbb{P}^{n}$, and $G$ a Green current for $\mathcal{X}$, that is a $(p-1, p-1)$ current on $\mathbb{P}^{n}(\mathbb{C})$ such that $d d^{c}(G)+\delta_{X}$ is a smooth form) by the subgroup generated by elements $(0, \partial u+\bar{\partial} v)$, or $\left(\operatorname{div}(f),-i_{*} \ln |f|^{2}\right), f$ being some element in $\mathbb{Q}(\mathcal{Y})^{*}$, where $\mathcal{Y}$ is an irreducible codimension $p-1$ cycle in $\mathbb{P}^{n}$ and $i$ the inclusion $\mathcal{Y} \longrightarrow \mathcal{X}([\mathrm{BGS} 2], 3.3)$. When $\mathcal{X}(\mathbb{C})$ is defined by a regular sequence $\left(P_{1}, \ldots, P_{m}\right)$ of homogeneous polynomials in $\mathbb{Z}\left[X_{0}, \ldots, X_{n}\right]$, the $p$ Chow class which admits a representative $(\mathcal{X}, T)$, where $T$ satisfies $(13)$ and is orthogonal to harmonic forms, plays an important role in the definition of the arithmetic height proposed for example by Faltings [Fa]. That is the reason why it seems to be interesting to solve directly (13) in terms of the generators $P_{1}, \ldots, P_{m}$. This can be done if one uses the meromorphic $\mathcal{D}^{\prime}\left(\mathbb{P}^{n}(\mathbb{C})\right)$-valued function

$$
\left(\lambda_{1}, \ldots, \lambda_{m}\right) \longrightarrow \prod_{j=1}^{m}\left(\frac{\left|P_{j}(X)\right|^{2}}{\|X\|^{2 \operatorname{deg}\left(P_{j}\right)}}\right)^{\lambda_{j}} .
$$

To construct such a solution for (13) (see $[\mathrm{Y}]$ ), the idea is to profit from the factorisation of the integration current over a complete intersection in some open domain $\omega$ in $\mathbb{C}^{n}$; this factorisation involves the multidimensional residue current, and can be written

$$
\delta_{\left\{f_{1}=\ldots=f_{m}=0\right\}}=\bar{\partial}(1 / f) \wedge d f_{1} \wedge \ldots \wedge d f_{m} .
$$

The analytic continuation of distributions appears when, using the basic ideas 
from $[\mathrm{BGY}]$ and $[\mathrm{BGVY}]$, one writes $(14)$ as

$$
\left\langle\delta_{\left\{f_{1}=\ldots=f_{m}=0\right\}}, \varphi\right\rangle=\left(\int\left(\bigwedge_{j=1}^{m} \bar{\partial}\left(\frac{\left|f_{j}\right|^{t_{j} \lambda}}{f_{j}}\right) \wedge d f_{j}\right) \wedge \varphi\right)_{\lambda=0}
$$

$\left(t=\left(t_{1}, \ldots, t_{m}\right) \in\left(\mathbb{R}^{+*}\right)^{n}\right.$ and $\varphi$ being a $(n-m, n-m)$ smooth test form in $\left.\omega\right)$.

Using such ideas, one can solve equation (13) in the complete intersection case as well as in the case when $P_{1}, \ldots, P_{m}$ define an irreducible cycle in $\mathbb{P}^{n}$ (the homogeneous ideal $\left(P_{1}, \ldots, P_{m}\right)$ being prime in $\left.\mathbb{Q}\left[X_{0}, \ldots, X_{n}\right]\right)$. There remains the crucial point of finding some analog (based on multiplications of distributions performed with meromorphic continuation) of the product

$$
\left(C H^{p}\right)^{\wedge}\left(\mathbb{P}^{n}\right) \times\left(C H^{p}\right)^{\wedge}\left(\mathbb{P}^{n}\right) \longrightarrow\left(\left(C H^{p+q}\right)^{\wedge}\left(\mathbb{P}^{n}\right)\right)_{\mathbb{Q}}
$$

introduced by Gillet-Soulé. This would provide some explicit computation for the height (in the sense of Faltings) which is defined as

$$
h(\mathcal{X})=\ln \left(\text { order of } \Gamma\left(Z_{0}, \mathcal{O}_{Z_{0}}\right)\right)+\frac{1}{2} \int_{\mathbb{P}^{n}(\mathbb{C})} G_{n}
$$

where $\left(Z_{0}, G_{n}\right)$ is any representative in the $n+1$ Chow group of

$$
\widehat{\mathcal{X}} \cdot\left(\widehat{c}_{1}\left(\mathbb{P}^{n}\right)\right)^{n+1-p}
$$

(where $\widehat{c}_{1}\left(\mathbb{P}^{n}\right)$ is the Chow class of any pair $\left(\operatorname{div}(L),-\ln |L|^{2}\right), L$ being any generic hyperplane in $\left.\mathbb{P}^{n}\right)$. Unfortunately, the only height which is computable at this point (since the work of Stoll [St]) is the height of a linear subspace in $\mathbb{P}^{n}$.

We tried to emphasize in this conclusion (as in this whole survey) the role of multidimensional residues in intersection or division problems in algebraic geometry. If anything about degrees estimates seems to be well understood (apart from questions related to Briançon-Skoda effectiveness, as seen in section 2), a lot of questions remain about size estimates where the arithmetic nature of the problem is involved. The roles of algebraic geometry and complex analysis appear to be complementary.

\section{References}

[Ai] L. A. Aizenberg and A. P. Yuzhakov, Integral Representation and Residues in Multidimensional Complex Analysis, Amer. Math. Soc., Providence, 1983.

[Am] F. Amoroso, Bounds for the degrees in the membership test for a polynomial ideal, Acta Arith. 56 (1990), 19-24.

[Am1] -, Tests d'appartenance d'après un théorème de J. Kollar, C. R. Acad. Sci. Paris Sér. I 309 (1989), 691-694.

[Am2] —, Membership problem, in: Diophantine Approximations and Transcendental Numbers, Luminy 1990, P. Philippon (ed.), Walter de Gruyter, Berlin, 1992, 15-37.

[Am3] - On a conjecture of C. Berenstein and A. Yger, in: Proc. MEGA 94, Progr. Math., Birkhäuser, to appear. 
[Am4] F. Amoroso, The membership problem for smooth ideals, Publ. Math. Univ. P. et M. Curie, to appear.

[Am5] - The membership problem for "almost" complete intersection ideals, preprint.

[At] M. F. Atiyah, Resolution of singularities and division of distributions, Comm. Pure Appl. Math. 23 (1970), 145-150.

[BaS] D. Bayer and M. Stillman, On the complexity of computing syzygies, J. Symbolic Comp. 6 (1988), 135-147.

[BGVY] C. A. Berenstein, R. Gay, A. Vidras and A. Yger, Residue Currents and Bézout Identities, Progr. Math. 114, Birkhäuser, 1993.

[BT] C. A. Berenstein and B. A. Taylor, Interpolation problems in $\mathbb{C}^{n}$ with applications to harmonic analysis, J. Analyse Math. 38 (1980), 188-254.

[BY1] C. A. Berenstein and A. Yger, Bounds for the degrees in the division problem, Michigan Math. J. 37 (1990), 26-44.

[BY2] -, 一, Effective Bézout identities in $\mathbb{Q}\left[z_{1}, \ldots, z_{n}\right]$, Acta Math. 166 (1991), 69-120.

[BY3] -, 一, Une formule de Jacobi et ses conséquences, Ann. Sci. Ecole. Norm. Sup. 24 (1991), 363-377.

[BY4] - - - Formules de représentation intégrale et problèmes de division, in: Diophantine Approximations and Transcendental Numbers, P. Philippon (ed.), Walter de Gruyter Berlin, 1992, 15-37.

[BY5] - , - Bounds for the division problem, in: Proc. Sympos. Pure Math. 52, part 1, Amer. Math. Soc., 1991, 22-28.

[BGS1] J. B. Bost, H. Gillet et C. Soulé, Un analogue arithmétique du théorème de Bézout, C. R. Acad. Sci. Paris Sér. I 312 (1991), 845-848.

[BGS2] - - - - Heights of projective varieties and positive Green forms, J. Amer. Math. Soc. 7 (1994), 903-1027.

[Br1] W. D. Brownawell, Bounds for the degrees in the Nullstellensatz, Ann. of Math. 126 (1987), 577-591.

[Br2] —, Local diophantine Nullstellen inequalities, J. Amer. Math. Soc. 1 (1988), 311-322.

[Br3] -, Distance to common zeros and lower bounds for polynomials, in: Diophantine Approximations and Transcendental Numbers, Luminy 1990, P. Philippon (ed.), Walter de Gruyter, Berlin, 1992, 51-64.

[Br4] -, A prime power version of the Nullstellensatz, Michigan Math. J., to appear.

[Elk1] M. Elkadi, Une version effective du théorème de Briançon-Skoda dans le cas algébrique discret, Acta Arith. 66 (1994), 201-220.

[Elk2] _, Bornes pour les degrés et les hauteurs dans le problème de division, Michigan Math. J., to appear.

[Fa] G. Faltings, Diophantine approximation on Abelian varieties, Ann. of Math. 133 (1991), 549-576.

[GH] P. Griffiths and J. Harris, Principles of Algebraic Geometry, Wiley-Interscience, New York, 1978.

[GS] H. Gillet and C. Soulé, Arithmetic intersection theory, Publ. Math. I.H.E.S. 72 (1990), 93-174.

[HP] W. V. D. Hodge and D. Pedoe, An Introduction to Algebraic Geometry, 3 Volumes, Cambridge University Press, Cambridge, 1952.

[Jac] C. G. J. Jacobi, Theoremata nova algebraica circa systema duarum aequationum inter duas variabiles propositarum, in: Gesammelte Werke, Band III, 285-294.

[Ko] J. Kollár, Sharp effective Nullstellensatz, J. Amer. Math. Soc. 1 (1988), 963-975.

[Ky] A. M. Kytmanov, A transformation formula for Grothendieck residues and some of its applications, Siberian Math. J. 1989, 495-499.

[LT] J. Lipman and B. Teissier, Pseudorational local rings and a theorem of BriançonSkoda about integral closures of ideals, Michigan Math. J. 28 (1981), 97-115. 
[Mac] F. S. Macaulay, The Algebraic Theory of Modular Systems, Cambridge University Press, Cambridge, 1916.

[MaM] E. Mayr and A. Meyer, The complexity of the word problem for commutative semigroups and polynomial ideals, Adv. in Math. 127 (1988), 305-329.

[Ph1] P. Philippon, Critères pour l'indépendance algébrique, Publ. Math. I.H.E.S. 64 (1986), 5-52.

[Ph2] -, A propos du texte de W.D. Brownawell: "Bounds for the degrees in the Nullstellensatz", Ann. of Math. 127 (1988), 367-371.

[Ph3] -, Dénominateurs dans le théorème des zéros de Hilbert, Acta Arith. 58 (1991), $1-25$.

[Ph4] —, Sur des hauteurs alternatives I, Math. Ann. 289 (1991), 255-283.

[Sk] H. Skoda, Application des techniques $L^{2}$ à la théorie des idéaux d'une algèbre de fonctions holomorphes avec poids, Ann. Sci. Ecole. Norm. Sup. 5 (1972), 545-579.

[St] W. Stoll, About the value distribution of holomorphic maps into projective space, Acta Math. 123 (1969), 83-114.

[Ts] A. Tsikh, Multidimensional Residues and Their Applications, Transl. Math. Monographs, Amer. Math. Soc., 1992.

[Weil] A. Weil, L'intégrale de Cauchy et les fonctions de plusieurs variables, Math. Ann. 111 (1935), 178-182.

[Y] A. Yger, Résidus, courants résiduels et courants de Green, in: Actes du Colloque de Géométrie Analytique, Paris VII, 1992, Travaux en Cours, Hermann, Paris, to appear. 